\title{
Translational significance of Nodal, Cripto-1 and Notch4 in adult nevi
}

\author{
LUIGI STRIZZI $^{1-3}$, NAIRA V. MARGARYAN ${ }^{1}$, PEDRAM GERAMI ${ }^{4,5}$, ZAHRA HAGHIGHAT $^{4,5}$, PAUL W. HARMS ${ }^{6}$, \\ GABRIELE MADONNA $^{7}$, GERARDO BOTTI ${ }^{8}$, PAOLO A. ASCIERTO ${ }^{7}$ and MARY J.C. HENDRIX ${ }^{1,3,5}$ \\ ${ }^{1}$ Cancer Biology and Epigenomics Program, Stanley Manne Children's Research Institute, Ann \& Robert H. Lurie \\ Children's Hospital of Chicago; ${ }^{2}$ Department of Pathology; ${ }^{3}$ Robert H. Lurie Comprehensive Cancer Center; \\ ${ }^{4}$ Department of Dermatology, Northwestern University Feinberg School of Medicine; ${ }^{5}$ Northwestern University Feinberg \\ School of Medicine, Chicago, IL 60611, USA; ${ }^{6}$ Department of Pathology, University of Michigan Medical School, Ann Arbor, \\ MI 48109, USA; ${ }^{7}$ Melanoma, Cancer Immunotherapy and Innovative Therapy Unit; ${ }^{8}$ Department of Pathology, \\ Istituto Nazionale Tumori Fondazione Pascale, 80131 Napoli, Italy
}

Received November 2, 2015; Accepted May 24, 2016

DOI: $10.3892 / 01.2016 .4755$

\begin{abstract}
The TGF- $\beta$ associated growth factor Nodal is highly expressed in aggressive metastatic melanoma. Determining the risk for melanomagenesis from Nodal expression in nevi prior to the development of melanoma may be useful for both the screening and prevention of melanoma. Tissue sections of human adult nevi with or without a history of melanoma were stained by immunohistochemistry (IHC) for Nodal, the Nodal co-receptor Cripto-1, and Notch4, which have previously been shown to be associated with Nodal expression in melanoma. The degree of Nodal, Cripto-1 and Notch4 staining was scored and correlated with available clinical data. Median IHC scores for Nodal, Cripto-1 and Notch4 expression were significantly higher in nevi removed from patients who eventually developed melanoma compared with nevi from patients with no history of melanoma. In addition, the degree of Nodal expression in nevi from patients who eventually developed melanoma correlated significantly with the Breslow depth of the melanoma. Expression of Nodal and components of its signaling pathway in nevi may represent a biomarker for selecting a unique subset of patients requiring increased surveillance for screening and prevention of melanoma.
\end{abstract}

Correspondence to: Dr Luigi Strizzi or Dr Mary J.C. Hendrix, Cancer Biology and Epigenomics Program, Stanley Manne Children's Research Institute, Ann \& Robert H. Lurie Children's Hospital of Chicago, Northwestern University Feinberg School of Medicine, 225 East Chicago Ave, Box 222, Chicago, IL 60611, USA E-mail: 1strizzi@luriechildrens.org

E-mail:m-hendrix@northwestern.edu

Key words: Nodal, Cripto-1, Notch4, immunohistochemistry, nevus, biomarker, screening, melanoma

\section{Introduction}

Previous work has determined that aggressive melanomas express Nodal, an embryonic morphogen belonging to the TGF- $\beta$ family of growth factors (1). It was demonstrated that Nodal expression increases during the progression of melanoma, from superficial, radial growth phase to deeper, vertical growth phase to advanced stage, metastatic melanoma (1). A subsequent report confirmed initial observations of lower Nodal expression in superficial melanoma compared to robust expression in deep melanoma and metastatic melanoma (2). Furthermore, it has been demonstrated that normal and dysplastic nevi expressed lower levels of Nodal (2). A well-known risk factor for melanoma is the number and types of melanocytic nevi, indicating that these may represent precursor lesions to melanoma (3). In fact, patients diagnosed with familial dysplastic nevi syndrome who exhibit a large number of melanocytic nevi, sometimes $>50$, have shown an increased risk for developing melanoma (4). It is desirable, therefore, to characterize the molecular profile of excised nevi and determine unique expression pattern(s), which may facilitate screening of patients with an increased likelihood for developing melanoma.

Since Nodal is a secreted molecule, it may have the potential for paracrine effects by influencing the homeostasis of cells that surround or are distant to the secretory source of this powerful morphogen. This is in agreement with the phenomenon known as 'field cancerization' whereby abnormalities, such as genetic or epigenetic alterations or changes in growth factor and receptor expression profiles, have been documented in histologically normal tissues distant from areas of overt cancer (5). Given the positive 'feed-forward' inductive characteristics of Nodal signaling (6), we hypothesize that Nodal secreted from a melanoma induce its own expression in otherwise nonmalignant nevi located in regional or distant sites causing phenotypic changes in these benign lesions, which may lead to increased propensity of these lesions towards malignant transformation. In this retrospective 
study, the immunohistochemical expression patterns of Nodal were determined, together with Cripto-1, the co-receptor for Nodal (7), and Notch4, a stem cell-associated molecule with two RBPJ binding sites on the Nodal gene shown to be associated with Nodal expression in melanoma (8). These findings were correlated with available clinical data, including history of subsequent melanoma development.

\section{Materials and methods}

De-identified slides with tissue sections of human malignant and nonmalignant melanocytic lesions for immunohistochemistry (IHC) were collected in compliance with Institutional Review Board ethics committee approval of study design and protocols for patient consent from Lurie Children's Hospital, Northwestern University, Chicago, IL, USA (approval no. 2010-14126), University of Michigan, Ann Arbor, MI, USA (approval no. HUM00045834) and Istituto Nazionale Tumori (INT) Fondazione Pascale, Naples, Italy (approval no. DMTMT-OMTI-34-2011). Slides from a total of 225 individual cases were collected from the following sources: University of Michigan (nonmalignant negative history of melanoma, $n=12$; nonmalignant positive history of melanoma, $\mathrm{n}=16$; malignant melanoma, $\mathrm{N}=33$ ); INT Fondazione Pascale (nonmalignant negative history of melanoma, $n=65$ ); histological slides purchased from Northwestern University Department of Dermatology Tissue Core (nonmalignant negative history of melanoma $n=50$; nonmalignant positive history of melanoma, $n=49$ ). The de-identified slides were stained by IHC for Nodal, Cripto-1 and Notch4, following the procedure previously described (8). Briefly, following antigen retrieval and blocking steps, sections were incubated in primary antibody for $60 \mathrm{~min}$ [mouse monoclonal anti-Nodal (1:200 dilution; cat no. ab55676; Abcam, Cambridge, MA, USA); rabbit polyclonal anti-Notch4 (1:80 dilution; cat no. sc-5594; Santa Cruz Biotechnology, Inc., Dallas, TX, USA); rabbit polyclonal anti-Cripto-1 (1:1500 dilution; cat no. 600-401-997; Rockland Scientific International Inc., Limerick, PA, USA)], followed by ready-to-use anti-rabbit (cat no. GR602H) or anti-mouse (cat no. GM601H) biotinylated secondary antibody (Biocare Medical Inc., Concord, CA, USA), and then streptavidin-horseradish peroxidase (ThermoFisher Scientific Inc., Waltham, MA, USA). Color was developed with 3,3'-diaminobenzidine substrate (ThermoFisher Scientific Inc.) and sections were counterstained with hematoxylin (Biocare Medical Inc.). As a negative control, adjacent serial sections were incubated with species appropriate irrelevant IgG (Jackson ImmunoResearch Labs) at the same concentration as primary antibodies. The quality and intensity of staining were analyzed and scored at low power and high power in order to calculate an Index Score (IS), as previously described (2). Student's $t$-test, median test and Pearson correlation were used for statistical analyses of the appropriate data. $\mathrm{P}<0.05$ was considered to indicate a statistically significant difference.

\section{Results}

The IHC results demonstrated a broad range of staining intensities for Nodal, Cripto-1 and Notch4 (Fig. 1). After IHC intensity scores (IS) were assigned, cases were grouped either

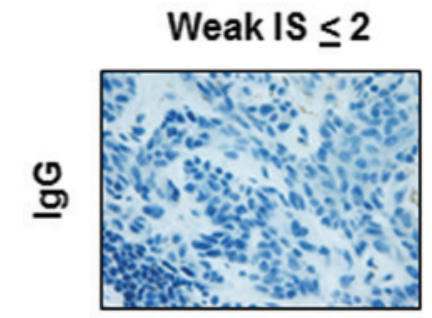

Strong IS 6 - 9
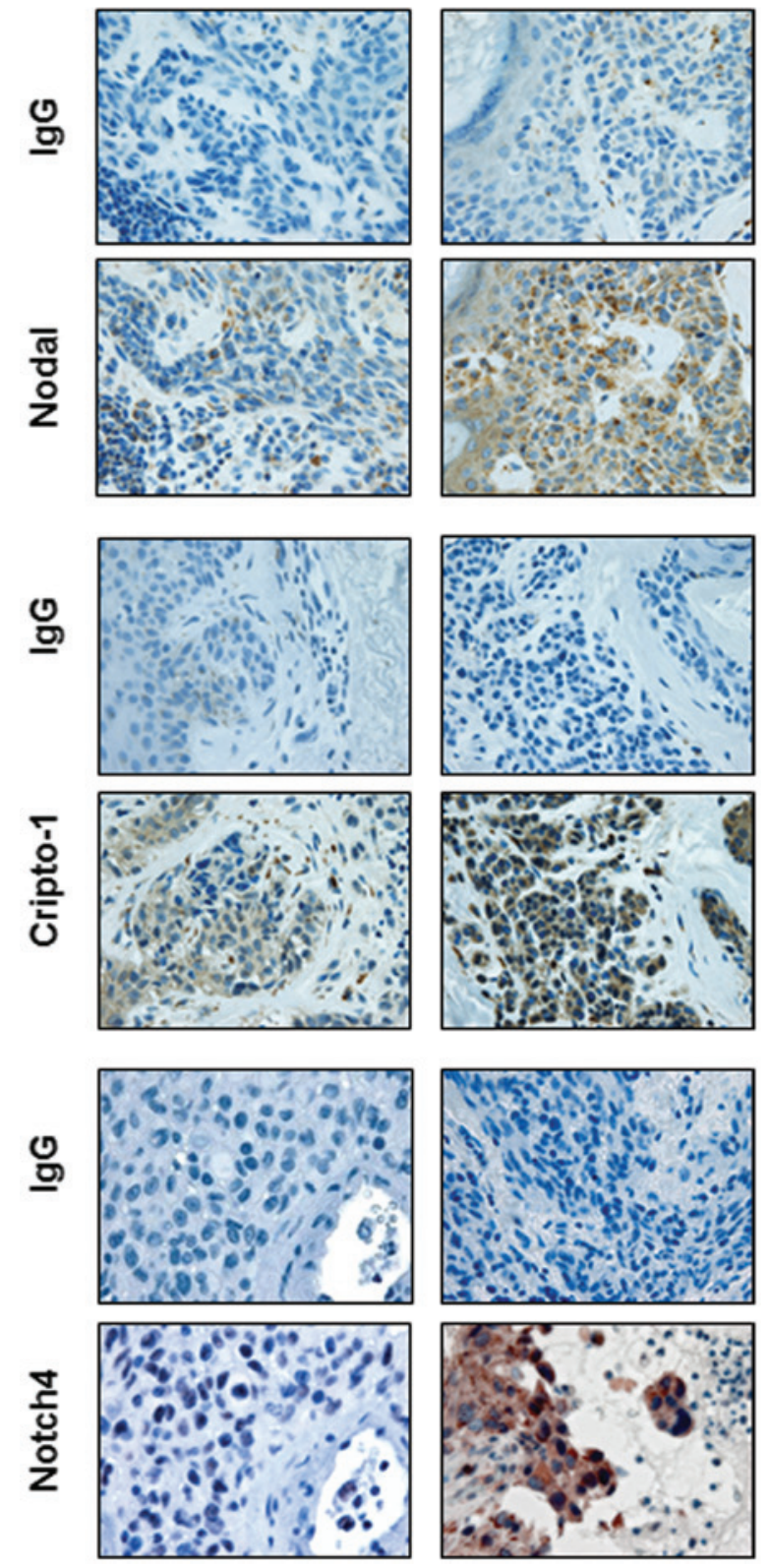

Figure 1. Immunohistochemistry results. Representative images of immunohistochemistry staining results showing weak and strong staining for Nodal, Cripto-1 and Notch4 in nevi. IS, index score.

as nevi from patients with no history of melanoma (negative $\mathrm{Hx}$ of melanoma) or nevi from patients that subsequently developed melanoma (positive Hx of melanoma). Mean IHC IS were calculated for Nodal, Cripto-1 and Notch4 and overall comparative analysis was performed to determine differences in expression levels among 4 groups for each marker, as summarized in Table I. The mean level for Nodal IS progressively increased from nevi with a negative history of melanoma to nevi with a positive history of melanoma to cutaneous melanoma to metastatic melanoma. The level of Nodal IS was significantly different among these groups (Fig. 2: all $\mathrm{P}<0.05$ versus negative history of melanoma). The same trend of progressive increase from nevi with a negative history of melanoma to nevi with a positive history of melanoma to cutaneous melanoma to metastatic melanoma was observed for Notch4 IS with a statistically 
Table I. Results of intensity of immunohistochemistry staining for Nodal, Cripto-1 and Notch4, represented as mean index score, in nevi from patients with a negative or positive history of melanoma, and cutaneous and metastatic melanoma

\begin{tabular}{|c|c|c|c|}
\hline Gene and type of lesion & Mean IS & $\mathrm{N}$ & P-value \\
\hline \multicolumn{4}{|l|}{ Nodal } \\
\hline Nevi, negative $\mathrm{Hx}$ of melanoma & $3.14+/-2.1$ & 129 & \\
\hline Nevi, positive Hx of melanoma & $4.77+/-2.45$ & 61 & $<0.01$ \\
\hline Cutaneous melanoma & $5.42+/-2.17$ & 12 & $<0.01$ \\
\hline Metastatic melanoma & $7.52+/-3.57$ & 21 & $<0.01$ \\
\hline \multicolumn{4}{|l|}{ Cripto-1 } \\
\hline Nevi, negative Hx of melanoma & $3.38+/-2.65$ & 128 & \\
\hline Nevi, positive Hx of melanoma & $4.3+/-2.98$ & 61 & 0.03 \\
\hline Cutaneous melanoma & $2.17+/-2.41$ & 12 & NS \\
\hline Metastatic melanoma & $3.57+/-3.03$ & 21 & NS \\
\hline \multicolumn{4}{|l|}{ Notch4 } \\
\hline Nevi, negative Hx of melanoma & $1.55+/-1.96$ & 121 & \\
\hline Nevi, positive Hx of melanoma & $2.08+/-1.87$ & 61 & NS \\
\hline Cutaneous melanoma & $2.58+/-2.71$ & 12 & NS \\
\hline Metastatic melanoma & $3.81+/-3.03$ & 21 & $<0.01$ \\
\hline
\end{tabular}

${ }^{\mathrm{a}} \mathrm{t}$-test vs. nevi with negative Hx melanoma. IS, index score; Hx, history.

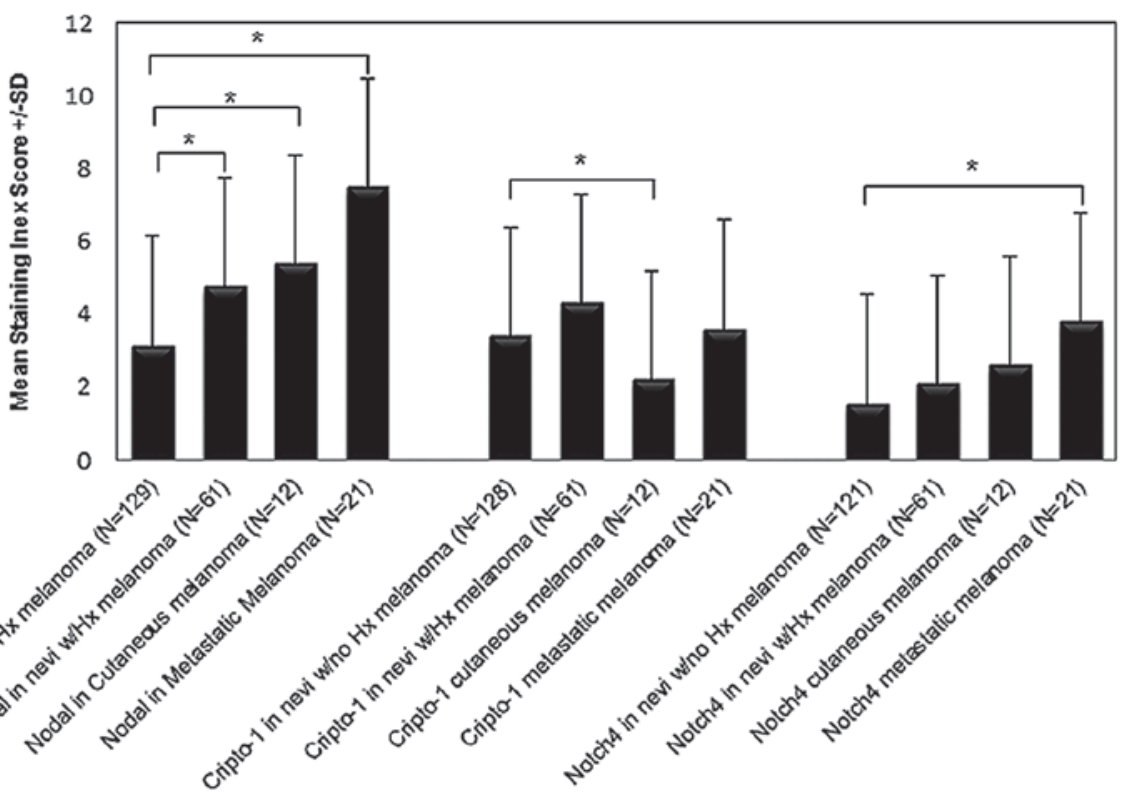

Figure 2. Immunohistochemistry IS. Histograms representative of mean immunohistochemistry IS for Nodal, Cripto-1 and Notch4 staining in nevi from patients with or without a $\mathrm{Hx}$ of melanoma and in cutaneous and metastatic melanoma ("P<0.05; $t$-test). IS, index score; Hx, history.

significant difference for the highest mean levels of Notch4 IS in the metastatic melanoma group (Fig. 2; $\mathrm{P}<0.05$ metastatic melanoma versus negative history of melanoma). Interestingly, mean Cripto-1 IS level was significantly greater only in the group of nevi from patients with a positive history of melanoma compared to the group of nevi from patients with a negative history of melanoma (Fig. 2; $\mathrm{P}<0.05$ ). There was no significant difference observed in the mean Cripto-1 IS between melanoma and the other groups. In addition, the cutaneous melanoma group showed the lowest mean Cripto-1 IS compared with the other groups (Fig. 2). Since calculation of mean values can be distorted by outliers, the median IS was also determined among the different groups for each marker. In fact, this analysis shows a significantly higher median IS for Nodal, Cripto-1 and Notch4 in the group of nevi from patients with a positive history of melanoma compared with the group of nevi from patients with no history of melanoma (Fig. 3).

Pearson's correlation analysis was performed to determine associations between Nodal, Cripto-1 and Notch4 expression levels for each group of nevi. Nodal levels did not correlate with 

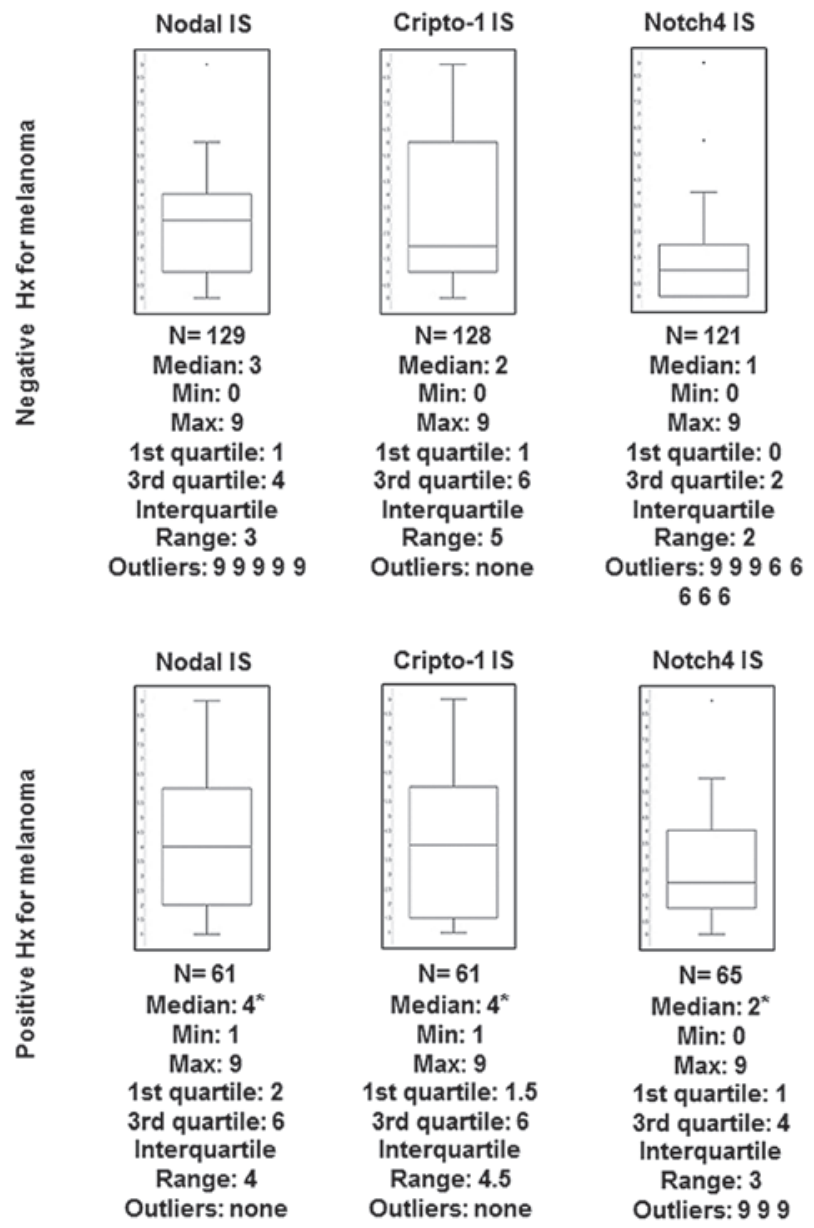

Figure 3. Median IS. Box plots representative of the distribution and median value of immunohistochemistry IS for Nodal, Cripto-1 and Notch4 staining in adult nevi (" $\mathrm{P}<0.05$; median test). IS, index score; Hx, history.

Cripto-1 or Notch4 in either group of nevi (data not shown). Notably, a significant positive correlation was observed for Cripto-1 and Notch4 expression levels in the group of nevi from patients with no history of melanoma $\left(\mathrm{R}^{2}=0.041 ; \mathrm{P}=0.03\right.$; $\mathrm{n}=121)$ and in the group of nevi from patients with a positive history of melanoma ( $\left.\mathrm{R}^{2}=0.141 ; \mathrm{P}=0.003 ; \mathrm{n}=61\right)$ (Fig. 4). In the group of nevi from patients with a positive history of melanoma, no significant correlation was observed between IS for Nodal, Cripto-1 or Notch4 and patient age, time elapsed between surgical removal of the nevus and diagnosis of melanoma, and anatomical site (data not shown). When analyzing high Nodal expression and anatomical sites in the group of nevi from female patients that subsequently developed melanoma, the highest Nodal IS (9) for both the nevi and the subsequent melanomas was predominantly associated with lesions located on the posterior torso (Fig. 5). In contrast, male patients showed the highest Nodal IS (9) for both the nevi and the subsequent melanomas in lesions located on the limbs (Fig. 5).

Finally, in patients with a positive history of melanoma, a significant positive correlation was observed between Nodal IS in the preceding nevus and Breslow depth in the subsequent melanomas $\left(\mathrm{R}^{2}=0.1067 ; \mathrm{P}=0.02 ; \mathrm{n}=48\right)$ (Fig. 6A). The same significant positive correlation was found between Nodal IS and Breslow depth in the cutaneous melanoma cases $\left(R^{2}=0.44\right.$; $\mathrm{P}=0.02 ; \mathrm{n}=12$ ) (Fig. 6B).

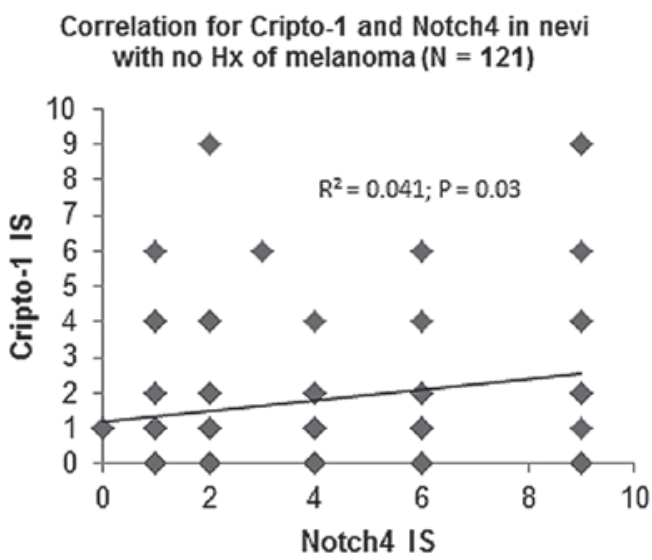

\section{Correlation for Cripto-1 and Notch4 in nevi with $\mathrm{Hx}$ of melanoma $(\mathrm{N}=61)$}

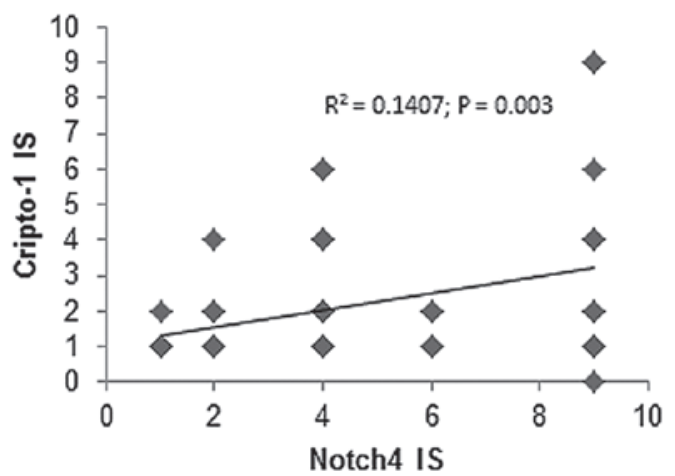

Figure 4. Correlation analyses. Linear correlation analysis between Nodal, Cripto-1 and Notch4 immunohistochmistry IS in nevi from patients with or without melanoma Hx. IS, index score; Hx, history.

\section{Discussion}

The present study demonstrated that there is a wide range of variability in the intensity of IHC staining of Nodal, Cripto-1 and Notch4 in nonmalignant melanocytic lesions regardless of whether or not the nevi were from patients with or without a history of melanoma, as reflected by the means of the IHC scores calculated for the different groups analyzed. Calculating the mean from a grading system of low to high expression levels, however, can be distorted by outlier cases having values at either end of the spectrum. Calculation of median levels with corresponding interquartile ranges provides an improved representation of the distribution of IHC scores for individual cases. In fact, this method showed a significantly higher median value for all three markers in the group of nevi from patients with a history of melanoma compared with nevi from patients with no melanoma history. However, on a case-by-case basis, no significant correlation was observed in IS between Nodal and Cripto-1 or Nodal and Notch4 for either group of nevi. This was unexpected since Cripto-1 is a well-established co-receptor for canonical Nodal signaling (7) and Notch 4 has been shown to be associated with Nodal expression in aggressive melanoma (8), and thus one would anticipate a relationship between these two protein markers. These data may indicate that either Nodal biological effects, assuming that nevi are responsive to Nodal, may be mediated independently of Cripto-1 or Notch4, or that Nodal's effect may 
Nodal IHC IS according to site of nevi in females $(\mathrm{N}=\mathbf{2 8})$

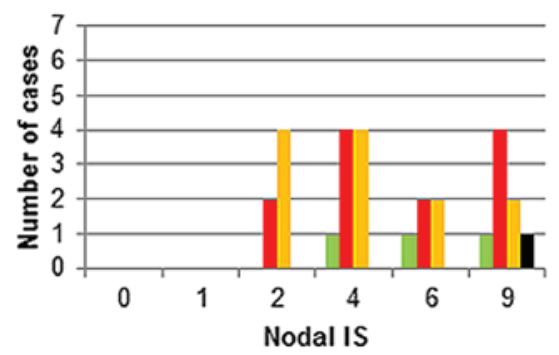

$\because$ Ant Torso $\square$ Post Torso $\backsim$ Limb $\square$ Head

Nodal IHC IS according to site of nevi in males $(\mathrm{N}=17)$

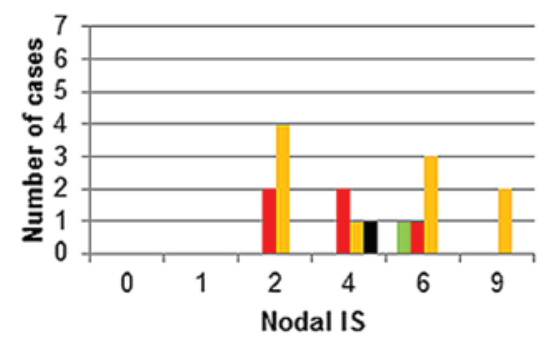

$\square$ Ant Torso $\backsim$ Post Torso $\square$ Limb $\backsim$ Head
Nodal IHC IS according to site of melanoma in females $(\mathrm{N}=\mathbf{2 6})$

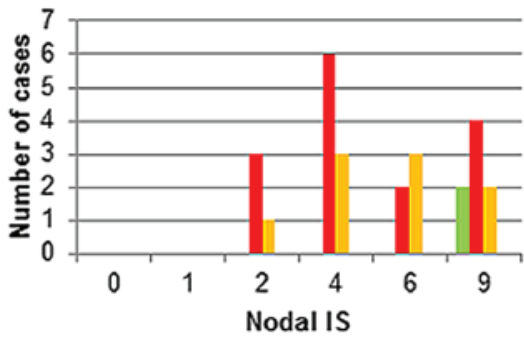

$\varpi$ Ant Torso $\backsim$ Post Torso $=$ Limb $\square$ Head

Nodal IHC IS according to site of melanoma in males $(\mathrm{N}=17)$

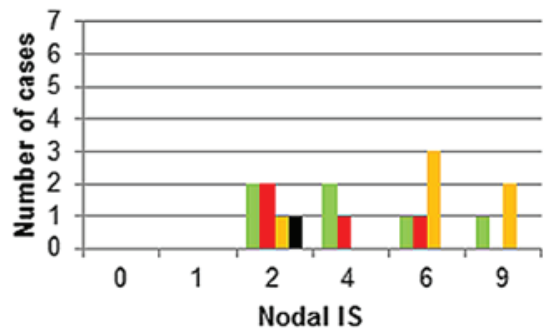

$\because$ Ant Torso $\backsim$ Post Torso $\square$ Limb $\square$ Head

Figure 5. Anatomical site of Nevus and Nodal expression. Nodal immunohistochemistry IS shown for nevi from patients with a positive history of melanoma and for the melanoma that subsequently developed according to anatomical sites and gender. IS, index score.

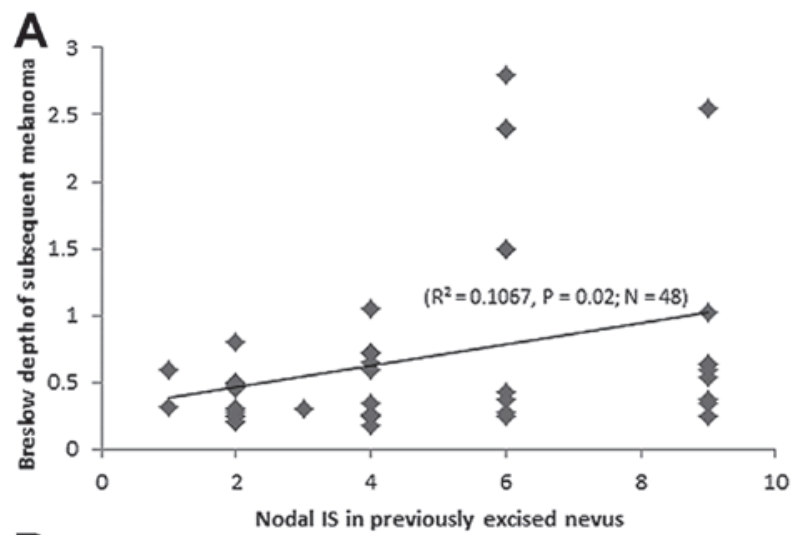

B

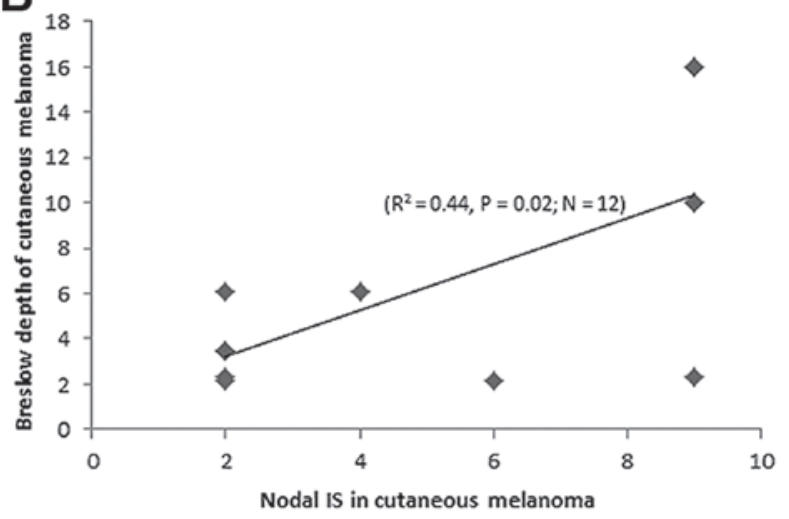

Figure 6. Nodal expression and melanoma depth. (A) Correlation between Nodal immunohistochemistry IS in nevi removed from patients prior to a diagnosis of melanoma and the Breslow depth of their melanomas that subsequently developed. (B) Correlation between Nodal IS and Breslow depth in cutaneous melanoma. IS, index score; Hx, history. become relevant only when Cripto- 1 and Notch4 reach critical levels. In fact, Nodal, Cripto-1 and Notch4 have been shown to reach significantly high expression levels in advanced stage melanoma $(8,9)$. Similarly, the data in the present study also show that Nodal, Cripto-1 and Notch4 reach higher levels in metastatic melanoma. Notably, a significant correlation was not observed between Cripto-1 and Notch4 expression in nevi regardless of the previous history of melanoma. Indeed, an earlier study demonstrated the close association between Cripto-1 and all 4 Notch proteins by describing the role for Cripto-1 in facilitating the post-translational maturation of Notch receptors (10). Perhaps this maturation process must occur in these nevi for Nodal to exert a more effective biological role. Further studies are required to determine the combined roles of Cripto-1 and Notch4 during melanomagenesis and how this may relate to Nodal expression and function in melanoma.

Since the nevi in the present study were surgically excised, a direct association between the high levels of Nodal, Cripto-1 and Notch4 and potential melanomagenesis in the excised nevus cannot be experimentally established. Nevertheless, a proportion of these patients eventually developed a melanoma following excision of the nevus, i.e. in the group of patients with a positive history of melanoma. Perhaps in this group, the melanomas may have developed from the malignant transformation of other high Nodal expressing nevi that were not yet excised. Alternatively, high Nodal expressing melanomas may secrete a level of Nodal capable of inducing additional Nodal expression in other nevi at locoregional sites in the same patient, since feedforward autoinduction from paracrine 
signaling is one of several established mechanisms underlying Nodal's biological effects $(6,11)$. In fact, in the group of nevi from patients that subsequently developed melanoma, the majority of these nevi showing highest Nodal expression (i.e. posterior torso) were from the same anatomical sites in which the melanoma subsequently developed-in both female and male patients. Perhaps the surrounding melanocytic cells/lesions in the posterior torso may have become more responsive to Nodal as a consequence of accumulated effects of other melanogenic factors, such as exposure to UV radiation. In fact, the posterior torso is a common site in both men and women for increased sun exposure. This also raises the possibility of a potential dosage effect of Nodal whereby, more Nodal would presumably exert more autoinductive effects. To this regard, it would be interesting to study the circulating Nodal levels and number of nevi to determine whether higher levels of Nodal can be detected in patients with increased numbers of nevi. Unfortunately, this data was not available since an exact count of all nevi present in each patient was not performed during whole body inspection prior to excision of the nevus. Notably, in the group of nevi from patients with a prior history of melanoma, for which accompanying Breslow depths were available for the melanomas that subsequently developed, a significant positive correlation was identified between Nodal expression in those nevi and thicker melanomas. These observations suggest that high Nodal expression in nevi may have the potential to predict approximate anatomical site and aggressiveness of a subsequent melanoma. No other clinical parameter, including patient age, anatomical site of the nevus or time elapsed between excision of the nevus and subsequent development of melanoma, correlated with any of the markers analyzed.

As in all retrospective studies such as this one, it is difficult to determine direct cause and effect. However, the significantly high levels of Nodal, Cripto-1 and Notch4 in nevi from patients that subsequently developed melanoma compared to the levels detected in the nevi from patients with no history of melanoma strongly suggests that Nodal signaling may serve a key role during melanoma development. Another limitation of the present study is the relatively small sample size due to the difficulty in collecting sufficient numbers of excised nevi from patients that subsequently develop melanoma, since the majority of these patients are lost to follow-up following nevi removal and the establishment of a benign diagnosis. Nevertheless, these data provide a strong evidence-based rationale for developing future, long term, and prospective follow-up studies of a large patient cohort with high Nodal expressing nevi, which will better determine the likelihood of these patients, with or without a history of melanoma, to subsequently develop a recurring or novel melanoma, respectively.

Nodal expression in melanoma is associated with aggressive, metastatic disease. The ability for early detection of Nodal in patients with benign melanocytic lesions could represent a powerful tool for screening and prevention of melanoma. The results of this study indicate that nevi with high Nodal expression are associated with the development of aggressive melanoma, suggesting that patients from whom these types of nevi are removed should be closely monitored for potential melanomagenesis.

\section{Acknowledgements}

The present study was supported by grants from the National Institutes of Health (Bethesda, MD, USA; grant nos. RO1CA121205 and R37CA59702) and H. Foundation and Dixon Translational Research Grants to Dr Mary J.C. Hendrix (Ann \& Robert H. Lurie Children's Hospital of Chicago, Northwestern University, Chicago, IL USA); and a grant from the American Cancer Society Institutional Research Grant (grant no. 93-037-18) to Dr Luigi Strizzi (Northwestern University).

\section{References}

1. Postovit LM, Margaryan NV, Seftor EA, Kirschmann DA, Lipavsky A, Wheaton WW, Abbott DE, Seftor RE and Hendrix MJ: Human embryonic stem cell microenvironment suppresses the tumorigenic phenotype of aggressive cancer cells. Proc Natl Acad Sci USA 105: 4329-4334, 2008.

2. Yu L, Harms PW, Pouryazdanparast P, Kim DS, Ma L and Fullen DR: Expression of the embryonic morphogen Nodal in cutaneous melanocytic lesions. Mod Pathol 23: 1209-1214, 2010.

3. Seykora J and Elder D: Dysplastic nevi and other risk markers for melanoma. Semin Oncol 23: 682-687, 1996.

4. Slade J, Marghoob AA, Salopek TG, Rigel DS, Kopf AW and Bart RS: Atypical mole syndrome: Risk factor for cutaneous malignant melanoma and implications for management. J Am Acad Dermatol 32: 479-494, 1995.

5. Dakubo GD, Jakupciak JP, Birch-Machin MA and Parr RL: Clinical implications and utility of field cancerization. Cancer Cell Int 7: 2, 2007.

6. D'Andrea D, Liguori GL, Le Good JA, Lonardo E, Andersson O, Constam DB, Persico MG and Minchiotti G: Cripto promotes A-P axis specification independently of its stimulatory effect on Nodal autoinduction. J Cell Biol 180: 597-605, 2008.

7. Shen MM: Nodal signaling: Developmental roles and regulation. Development 134: 1023-1034, 2007.

8. Hardy KM, Kirschmann DA, Seftor EA, Margaryan NV, Postovit LM, Strizzi L and Hendrix MJ: Regulation of the embryonic morphogen Nodal by Notch4 facilitates manifestation of the aggressive melanoma phenotype. Cancer Res 70: 10340-10350, 2010.

9. De Luca A, Lamura L, Strizzi L, Roma C, D'Antonio A, Margaryan N, Pirozzi G, Hsu MY, Botti G, Mari E, et al: Expression and functional role of CRIPTO-1 in cutaneous melanoma. Br J Cancer 105: 1030-1038, 2011.

10. Watanabe K, Nagaoka T, Lee JM, Bianco C, Gonzales M, Castro NP, Rangel MC, Sakamoto K, Sun Y, Callahan R and Salomon DS: Enhancement of Notch receptor maturation and signaling sensitivity by Cripto-1. J Cell Biol 187: 343-353, 2009.

11. Le Good JA, Joubin K, Giraldez AJ, Ben-Haim N, Beck S, Chen Y, Schier AF and Constam DB: Nodal stability determines signaling range. Curr Biol 15: 31-36, 2005. 